\title{
ISOMETRIC DILATIONS FOR INFINITE SEQUENCES OF NONCOMMUTING OPERATORS
}

\author{
GELU POPESCU
}

\begin{abstract}
This paper develops a dilation theory for $\left\{T_{n}\right\}_{n=1}^{\infty}$ an infinite sequence of noncommuting operators on a Hilbert space, when the matrix $\left[T_{1}, T_{2}\right.$, ...] is a contraction. A Wold decomposition for an infinite sequence of isometries with orthogonal final spaces and a minimal isometric dilation for $\left\{T_{n}\right\}_{n=1}^{\infty}$ are obtained. Some theorems on the geometric structure of the space of the minimal isometric dilation and some consequences are given. This results are used to extend the Sz.-Nagy-Foiaş lifting theorem to this noncommutative setting.
\end{abstract}

This paper is a continuation of [5] and develops a dilation theory for an infinite sequence $\left\{T_{n}\right\}_{n=1}^{\infty}$ of noncommuting operators on a Hilbert space $\mathscr{H}$ when $\sum_{n=1}^{\infty} T_{n} T_{n}^{*} \leq I_{\mathscr{H}}\left(I_{\mathscr{H}}\right.$ is the identity on $\left.\mathscr{H}\right)$.

Many of the results and techniques in dilation theory for one operator [8] and also for two operators $[3,4]$ are extended to this setting.

First we extend Wold decomposition $[8,4]$ to the case of an infinite sequence $\left\{V_{n}\right\}_{n=1}^{\infty}$ of isometries with orthogonal final spaces.

In $\S 2$ we obtain a minimal isometric dilation for $\left\{T_{n}\right\}_{n=1}^{\infty}$ by extending the Schaffer construction in [6, 4]. Using these results we give some theorems on the geometric structure of the space of the minimal isometric dilation. Finally, we give some sufficient conditions on a sequence $\left\{T_{n}\right\}_{n=1}^{\infty}$ to be simultaneously quasi-similar to a sequence $\left\{R_{n}\right\}_{n=1}^{\infty}$ of isometries on a Hilbert space $\mathscr{K}$ with $\sum_{n=1}^{\infty} R_{n} R_{n}^{*}=I_{\mathscr{K}}$.

In $\S 3$ we use the above-mentioned theorems to obtain the Sz.-Nagy-Foias lifting theorem $[7,8,1,4]$ in our setting.

In a subsequent paper we will use the results of this paper for studying the "characteristic function" associated to a sequence $\left\{T_{n}\right\}_{n=1}^{\infty}$ with $\sum_{n=1}^{\infty} T_{n} T_{n}^{*} \leq$ $I_{\not{H}}$.

Throughout this paper $\Lambda$ stands for the set $\{1,2, \ldots, k\} \quad(k \in \mathbf{N})$ or the set $\mathbf{N}=\{1,2, \ldots\}$.

Received by the editors September 1, 1987.

1980 Mathematics Subject Classification (1985 Revision). Primary 47A20; Secondary 47A45.

Key words and phrases. Isometric dilation, Wold decomposition, lifting theorem. 
For every $n \in \mathbf{N}$ let $F(n, \Lambda)$ be the set of all functions from the set $\{1,2, \ldots, n\}$ to $\Lambda$ and

$$
\mathscr{F}=\bigcup_{n=0}^{\infty} F(n, \Lambda), \quad \text { where } F(0, \Lambda)=\{0\} .
$$

Let $\mathscr{H}$ be a Hilbert space and $\mathscr{V}=\left\{V_{\lambda}\right\}_{\lambda \in \Lambda}$ be a sequence of isometries on $\mathscr{H}$. For any $f \in F(n, \Lambda)$ we denote by $V_{f}$ the product $V_{f(1)} V_{f(2)} \cdots V_{f(n)}$ and $V_{0}=I_{\mathscr{H}}$.

A subspace $\mathscr{L} \subset \mathscr{H}$ will be called wandering for the sequence $\mathscr{V}$ if for any distinct functions $f, g \in \mathscr{F}$ we have

$$
V_{f} \mathscr{L} \perp V_{g} \mathscr{L} \quad(\perp \text { means orthogonal). }
$$

In this case we can form the orthogonal sum

$$
M_{\mathscr{F}}(\mathscr{L})=\bigoplus_{f \in \mathscr{F}} V_{f} \mathscr{L}
$$

A sequence $\mathscr{V}=\left\{V_{\lambda}\right\}_{\lambda \in \Lambda}$ of isometries on $\mathscr{H}$ is called a $\Lambda$-orthogonal shift if there exists in $\mathscr{H}$ a subspace $\mathscr{L}$, which is wandering for $\mathscr{V}$ and such that $\mathscr{H}=M_{\mathscr{F}}(\mathscr{L})$.

This subspace $\mathscr{L}$ is uniquely determined by $\mathscr{V}$ : indeed we have $\mathscr{L}=$ $\mathscr{H} \ominus\left(\bigoplus_{\lambda \in \Lambda} V_{\lambda} \mathscr{H}\right)$. The dimension of $\mathscr{L}$ is called the multiplicity of the $\Lambda$ orthogonal shift. One can show, by an argument similar to the classical unilateral shift, that a $\Lambda$-orthogonal shift is determined up to unitary equivalence by its multiplicity. It is easy to see that for $\Lambda=\{1\}$ we find again the classical unilateral shift.

Let us make some simple remarks whose proofs will be omitted.

Remark 1.1. If $\mathscr{V}=\left\{V_{\lambda}\right\}_{\lambda \in \Lambda}$ is a $\Lambda$-orthogonal shift on $\mathscr{H}$, with the wandering subspace $\mathscr{L}$, then for any $n \in \mathbf{N}, \lambda \in \Lambda$ and $f \in F(n, \Lambda)$ we have

(a)

$$
V_{\lambda}^{*} V_{f}= \begin{cases}V_{f(2)} V_{f(3)} \cdots V_{f(n)} & \text { if } f(1)=\lambda, \\ 0 & \text { if } f(1) \neq \lambda,\end{cases}
$$

and $V_{\lambda}^{*} \ell=0 \quad(\ell \in \mathscr{L})$.

(b) $\sum_{\lambda \in \Lambda} V_{\lambda} V_{\lambda}^{*}+P_{\mathscr{L}}=I_{\mathscr{H}}$, where $P_{\mathscr{L}}$, stands for the orthogonal projection from $\mathscr{H}$ into $\mathscr{L}$.

Remark 1.2. If $\mathscr{V}=\left\{V_{\lambda}\right\}_{\lambda \in \Lambda}$ is a $\Lambda$-orthogonal shift on $\mathscr{H}$ then

(a) $\lim _{n \rightarrow \infty} \sum_{f \in F(n, \Lambda)}\left\|V_{f}^{*} h\right\|^{2}=0$, for any $h \in \mathscr{H}$.

(b) $V_{\lambda}^{* k} \rightarrow 0$ (strongly) as $k \rightarrow \infty$, for any $\lambda \in \Lambda$.

(c) There exists no nonzero reducing subspace $\mathscr{H}_{0} \subset \mathscr{H}$ for each $V_{\lambda} \quad(\lambda \in$ $\Lambda)$ such that $\left.\left(I_{\not{H}}-\sum_{\lambda \in \Lambda} V_{\lambda} V_{\lambda}^{*}\right)\right|_{\mathscr{H}_{0}}=0$.

Let us consider a model $\Lambda$-orthogonal shift. 
Form the Hilbert space

$$
l^{2}(\mathscr{F}, \mathscr{H})=\left\{\left(h_{f}\right)_{f \in \mathscr{F}} ; \sum_{f \in \mathscr{F}}\left\|h_{f}\right\|^{2}<\infty, h_{f} \in \mathscr{H}\right\} .
$$

We embed $\mathscr{H}$ in $l^{2}(\mathscr{F}, \mathscr{H})$ as a subspace, by identifying the element $h \in \mathscr{H}$ with the element $\left(h_{f}\right)_{f \in \mathscr{F}}$, where $h_{0}=h$ and $h_{f}=0$ for any $f \in \mathscr{F}, f \neq 0$.

For each $\lambda \in \Lambda$ we define the operator $S_{\lambda}$ on $l^{2}(\mathscr{F}, \mathscr{H})$ by $S_{\lambda}\left(\left(h_{f}\right)_{f \in \mathscr{F}}\right)=$ $\left(h_{g}^{\prime}\right)_{g \in \mathscr{F}}$, where $h_{0}^{\prime}=0$ and for $g \in F(n, \Lambda) \quad(n \geq 1)$

$$
h_{g}^{\prime}= \begin{cases}h_{0} \quad \text { if } g \in F(1, \Lambda) \text { and } g(1)=\lambda \\ h_{f} \quad \text { if } g \in F(n, \Lambda)(n \geq 2), f \in F(n-1, \Lambda) \text { and } g(1)=\lambda, \\ g(2)=f(1), g(3)=f(2), \ldots, g(n)=f(n-1), \\ 0 \quad \text { otherwise }\end{cases}
$$

It is easy to see that $\left\{S_{\lambda}\right\}_{\lambda \in \Lambda}$ is the $\Lambda$-orthogonal shift, acting on $l^{2}(\mathscr{F}, \mathscr{H})$, with the wandering subspace $\mathscr{H}$.

This model plays an important role in this paper. The following theorem is our version of Wold decomposition for a sequence of isometries.

Theorem 1.3. Let $\mathscr{V}=\left\{V_{\lambda}\right\}_{\lambda \in \Lambda}$ be a sequence of isometries on a Hilbert space $\mathscr{K}$ such that $\sum_{\lambda \in \Lambda} V_{\lambda} V_{\lambda}^{*} \leq I_{\mathscr{K}}$.

Then $\mathscr{K}$ decomposes into an orthogonal sum $\mathscr{K}=\mathscr{K}_{0} \oplus \mathscr{K}_{1}$ such that $\mathscr{K}_{0}$ and $\mathscr{K}_{1}$ reduce each operator $V_{\lambda}(\lambda \in \Lambda)$ and we have $\left.\left(I_{\mathscr{K}}-\sum_{\lambda \in \Lambda} V_{\lambda} V_{\lambda}^{*}\right)\right|_{\mathscr{H}_{1}}=0$ and $\left\{\left.V_{\lambda}\right|_{\mathscr{H}_{0}}\right\}_{\lambda \in \Lambda}$ is a $\Lambda$-orthogonal shift acting on $\mathscr{K}_{0}$.

This decomposition is uniquely determined, indeed we have

$$
\mathscr{K}_{1}=\bigcap_{n=0}^{\infty}\left(\bigoplus_{f \in F(n, \Lambda)} V_{f} \mathscr{K}\right)
$$

$\mathscr{K}_{0}=M_{\mathscr{F}}(\mathscr{L})$, where $\mathscr{L}=\mathscr{K} \ominus\left(\bigoplus_{\lambda \in \Lambda} V_{\lambda} \mathscr{K}\right)$.

Proof. It is easy to see that the subspace $\mathscr{L}=\mathscr{K} \ominus\left(\bigoplus_{\lambda \in \Lambda} V_{\lambda} \mathscr{K}\right)$ is wandering for $\mathscr{V}$.

Now let $\mathscr{K}_{0}=M_{\mathscr{F}}(\mathscr{L})$ and $\mathscr{K}_{1}^{\prime}=\mathscr{K} \ominus \mathscr{K}_{0}$. Observe that $k \in \mathscr{K}_{1}^{\prime}$ if and only if $k \perp \bigoplus_{f \in \mathscr{F}_{n}} V_{f} \mathscr{L}$ for every $n \in \mathbf{N}$, where $\mathscr{F}_{n}$ stands for $\bigcup_{k=0}^{n} F(k, \Lambda)$. 
We have

$$
\begin{aligned}
& \mathscr{L} \oplus\left(\bigoplus_{f \in F(1, \Lambda)} V_{f} \mathscr{L}\right) \oplus \oplus\left(\bigoplus_{g \in F(n, \Lambda)} V_{g} \mathscr{L}\right)=\left[\mathscr{K} \ominus\left(\bigoplus_{f \in F(1, \Lambda)} V_{f} \mathscr{K}\right)\right] \\
& \oplus {\left[\left(\bigoplus_{f \in F(1, \Lambda)} V_{f} \mathscr{K}\right) \ominus\left(\bigoplus_{f \in F(2, \Lambda)} V_{f} \mathscr{K}\right)\right] } \\
& \oplus \cdots \oplus\left[\left(\bigoplus_{f \in F(n, \Lambda)} V_{f} \mathscr{K}\right) \ominus\left(\underset{f \in F(n+1, \Lambda)}{\bigoplus} V_{f} \mathscr{K}\right)\right] \\
&=\mathscr{K} \ominus\left(\bigoplus_{f \in F(n+1, \Lambda)} V_{f} \mathscr{K}\right) .
\end{aligned}
$$

Thus $k \in \mathscr{K}_{1}^{\prime}$ if and only if $k \in \bigoplus_{f \in F(n+1, \Lambda)} V_{f} \mathscr{K}$ for every $n \in \mathbf{N}$. Since

$$
\bigoplus_{f \in F(n, \Lambda)} V_{f} \mathscr{K} \supset \bigoplus_{f \in F(n+1, \Lambda)} V_{f} \mathscr{K} \quad(n \in \mathbf{N})
$$

it follows that

$$
\mathscr{K}_{1}^{\prime}=\bigcap_{n=0}^{\infty}\left(\bigoplus_{f \in F(n, \Lambda)} V_{f} \mathscr{K}\right)=\mathscr{K}_{1} .
$$

Let us notice that

$$
\begin{aligned}
& V_{\lambda} \mathscr{K}_{1} \subset \bigcap_{n=0}^{\infty}\left(\bigoplus_{f \in F(n, \Lambda)} V_{\lambda} V_{f} \mathscr{K}\right) \subset \bigcap_{n=0}^{\infty}\left(\bigoplus_{g \in F(n+1, \Lambda)} V_{g} \mathscr{K}\right)=\mathscr{K}_{1}, \\
& V_{\lambda}^{*} \mathscr{K}_{1} \subset \bigcap_{n=1}^{\infty}\left(V_{\lambda}^{*}\left(\bigoplus_{\substack{g \in F(n, \Lambda) \\
g(1)=\lambda}} V_{g} \mathscr{K}\right)\right)=\bigcap_{n=1}^{\infty}\left(\underset{f \in F(n-1, \Lambda)}{\bigoplus} V_{f} \mathscr{K}\right)=\mathscr{K}_{1} .
\end{aligned}
$$

Therefore $\mathscr{K}_{1}$ reduces each $V_{\lambda}(\lambda \in \Lambda)$. Hence $\mathscr{K}_{0}$ also reduces each $V_{\lambda}$ $(\lambda \in \Lambda)$.

Since $\mathscr{K}_{1} \subset \bigoplus_{\lambda \in \Lambda} V_{\lambda} \mathscr{K}$ it follows that $\left.\left(I_{\mathscr{K}}-\sum_{\lambda \in \Lambda} V_{\lambda} V_{\lambda}^{*}\right)\right|_{\mathscr{K}_{1}}=0$. The fact that $\left\{\left.V_{\lambda}\right|_{\mathscr{Z}_{0}}\right\}_{\lambda \in \Lambda}$ is a $\Lambda$-orthogonal shift is obvious. The uniqueness of the decomposition follows by an argument similar to the classical Wold decomposition [8, Chapter I, Theorem 1.1]. The proof is completed.

Remark 1.4. The subspaces $\mathscr{K}_{0}, \mathscr{K}_{1}$ from Wold decomposition can be described as follows:

$$
\begin{aligned}
& \mathscr{K}_{0}=\left\{k \in \mathscr{K}: \lim _{n \rightarrow \infty} \sum_{f \in F(n, \Lambda)}\left\|V_{f}^{*} k\right\|^{2}=0\right\}, \\
& \mathscr{K}_{1}=\left\{k \in \mathscr{K}: \sum_{f \in F(n, \Lambda)}\left\|V_{f}^{*} k\right\|^{2}=\|k\|^{2} \text { for every } n \in \mathbf{N}\right\} .
\end{aligned}
$$


We call the sequence $\mathscr{V}=\left\{V_{\lambda}\right\}_{\lambda \in \Lambda}$ in Theorem 1.3 pure if $\mathscr{K}_{1}=0$, that is, if $\mathscr{V}$ is a $\Lambda$-orthogonal shift on $\mathscr{K}$.

Let $\mathscr{T}=\left\{T_{\lambda}\right\}_{\lambda \in \Lambda}$ a sequence of contractions on a Hilbert space $\mathscr{H}$ such that $\sum_{\lambda \in \Lambda} T_{\lambda} T_{\lambda}^{*} \leq I_{\mathscr{H}}$.

We say that a sequence $\mathscr{V}=\left\{V_{\lambda}\right\}_{\lambda \in \Lambda}$ of isometries on a Hilbert space $\mathscr{K} \supset$ $\mathscr{H}$ is a minimal isometric dilation of $\mathscr{T}$ if the following conditions hold:

(a) $\sum_{\lambda \in \Lambda} V_{\lambda} V_{\lambda}^{*} \leq I_{\mathscr{K}}$.

(b) $\mathscr{H}$ is invariant for each $V_{\lambda}^{*}(\lambda \in \Lambda)$ and $\left.V_{\lambda}^{*}\right|_{\mathscr{H}}=T_{\lambda}^{*}(\lambda \in \Lambda)$.

(c) $\mathscr{K}=\bigvee_{f \in \mathscr{F}} V_{f} \mathscr{H}$.

Let $D_{*}$ on $\mathscr{H}$ and $D$ on $\bigoplus_{\lambda \in \Lambda} \mathscr{H}_{\lambda}\left(\mathscr{H}_{\lambda}\right.$ is a copy of $\left.\mathscr{H}\right)$ be the positive operators uniquely defined by $D_{*}=\left(I_{\mathscr{H}}-\sum_{\lambda \in \Lambda} T_{\lambda} T_{\lambda}^{*}\right)^{1 / 2}$ and $D=D_{T}$, where $T$ stands for the matrix $\left[T_{1}, T_{2}, \ldots\right]$ and $D_{T}=\left(I-T^{*} T\right)^{1 / 2}$.

Let us denote $\mathscr{D}_{*}=\overline{D_{*} \mathscr{H}}$ and $\mathscr{D}=\overline{D\left(\bigoplus_{\lambda \in \Lambda} \mathscr{H}_{\lambda}\right)}$.

Theorem 2.1. For every sequence $\mathscr{T}=\left\{T_{\lambda}\right\}_{\lambda \in \Lambda}$ of noncommuting operators on a Hilbert space $\mathscr{H}$ such that $\sum_{\lambda \in \Lambda} T_{\lambda} T_{\lambda}^{*} \leq I_{\mathscr{H}}$, there exists a minimal isometric dilation $\mathscr{V}=\left\{V_{\lambda}\right\}_{\lambda \in \Lambda}$ on a Hilbert space $\mathscr{K} \supset \mathscr{H}$, which is uniquely determined up to an isomorphism.

Proof. Let us consider the Hilbert space $\mathscr{K}=\mathscr{H} \oplus l^{2}(\mathscr{F}, \mathscr{D})$. We embed $\mathscr{H}$ and $\mathscr{D}$ into $\mathscr{K}$ in a natural way. For each $\lambda \in \Lambda$ we define the isometry $V_{\lambda}: \mathscr{K} \rightarrow \mathscr{K}$ by the relation

$$
V_{\lambda}\left(h \oplus\left(d_{f}\right)_{f \in \mathscr{F}}\right)=T_{\lambda} h \oplus\left(D(\underbrace{0, \ldots, 0}_{\lambda-1 \text { times }}, h, 0, \ldots)+S_{\lambda}\left(d_{f}\right)_{f \in \mathscr{F}}\right)
$$

where $\left\{S_{\lambda}\right\}_{\lambda \in \Lambda}$ is $\Lambda$-orthogonal shift on $l^{2}(\mathscr{F}, \mathscr{D})$ (see $\S 1$ ).

Obviously, for any $\lambda, \mu \in \Lambda, \lambda \neq \mu$ we have range $S_{\lambda} \perp$ range $S_{\mu}$ and

$$
\left(T_{\mu}^{*} T_{\lambda} h, h^{\prime}\right)=-\left(D^{2}(\underbrace{0, \ldots, 0}_{\lambda-1 \text { times }}, h, 0, \ldots),(\underbrace{0, \ldots, 0}_{\mu-1 \text { times }}, h^{\prime}, 0, \ldots)\right) .
$$

Hence, taking into account (2.1), it follows that

$$
\text { range } V_{\lambda} \perp \text { range } V_{\mu} \quad(\lambda, \mu \in \Lambda, \lambda \neq \mu)
$$

therefore $\sum_{\lambda \in \Lambda} V_{\lambda} V_{\lambda}^{*} \leq I_{\mathscr{H}}$.

It is easy to show that $\mathscr{H}$ is invariant for each $V_{\lambda}^{*}(\lambda \in \Lambda)$ and $\left.V_{\lambda}^{*}\right|_{\mathscr{H}}=T_{\lambda}^{*}$ $(\lambda \in \Lambda)$.

Finally, we verify that $\mathscr{V}=\left\{V_{\lambda}\right\}_{\lambda \in \Lambda}$ is the minimal isometric dilation of $\mathscr{T}$.

Let $\mathscr{H}_{1}=\mathscr{H} \vee\left(\bigvee_{f \in F(1, \Lambda)} V_{f} \mathscr{H}\right)$ and

$$
\mathscr{H}_{n}=\mathscr{H}_{n-1} \vee\left(\bigvee_{f \in F(1, \Lambda)} V_{f} \mathscr{H}_{n-1}\right) \quad \text { if } n \geq 2
$$


It is easy to see that $\mathscr{H}_{1}=\mathscr{H} \oplus \mathscr{D}$ and

$$
\mathscr{H}_{n}=\mathscr{H} \oplus \mathscr{D} \oplus\left(\bigoplus_{f \in F(1, \Lambda)} S_{f} \mathscr{D}\right) \oplus \cdots \oplus\left(\bigoplus_{f \in F(n-1, \Lambda)} S_{f} \mathscr{D}\right) \quad \text { if } n \geq 2 .
$$

Clearly $\mathscr{H}_{n} \subset \mathscr{H}_{n+1}$ and we have

$$
\bigvee_{1}^{\infty} \mathscr{H}_{n}=\mathscr{H} \oplus M_{\mathscr{F}}(\mathscr{D})=\mathscr{H} \oplus l^{2}(\mathscr{F}, \mathscr{D})=\mathscr{K} .
$$

Therefore $\mathscr{H}=\bigvee_{f \in \mathscr{F}} V_{f} \mathscr{H}$.

Following Theorem 4.1 in [8, Chapter I] it is easy to show that the minimal isometric dilation $\mathscr{V}$ of $\mathscr{T}$ is unique up to a unitary operator. To be more precise, let $\mathscr{V}^{\prime}=\left\{V_{\lambda}^{\prime}\right\}_{\lambda \in \Lambda}$ be another minimal isometric dilation of $\mathscr{T}$, on a Hilbert space $\mathscr{K}^{\prime} \supset \mathscr{H}$. Then there exists a unitary operator $U: \mathscr{K} \rightarrow \mathscr{K}^{\prime}$ such that $V_{\lambda}^{\prime} U=U V_{\lambda}(\lambda \in \Lambda)$ and $U h=h$ for every $h \in \mathscr{H}$.

This completes the proof.

Remark 2.2. For each $\lambda \in \Lambda, \bar{V}_{\lambda}^{* n} \rightarrow 0$ (strongly) as $n \rightarrow \infty$ if and only if $T_{\lambda}^{* n} \rightarrow 0$ (strongly) as $n \rightarrow \infty$.

From this remark and Theorem 2.1 one can easily deduce Proposition 1.1 in [5].

The following is a generalization of [2] or Theorem 1.2 in [8, Chapter II].

Propostion 2.3. Let $\mathscr{V}=\left\{V_{\lambda}\right\}_{\lambda \in \Lambda}$ be the minimal isometric dilation of $\mathscr{T}=$ $\left\{T_{\lambda}\right\}_{\lambda \in \Lambda}$. Then $\mathscr{V}$ is pure if and only if

$$
\lim _{n \rightarrow \infty} \sum_{f \in F(n, \Lambda)}\left\|T_{f}^{*} h\right\|^{2}=0
$$

for any $h \in \mathscr{H}$.

Proof. Assume that $\mathscr{V}$ is pure. Then, by Theorem 1.3 it follows that $\mathscr{V}$ is a $\Lambda$-orthogonal shift on the space $\mathscr{K} \supset \mathscr{H}$ of the minimal isometric dilation of $\mathscr{T}$.

Taking into account Remark 1.2 and the fact that for each $f \in \mathscr{F},\left.V_{f}^{*}\right|_{\mathscr{H}}=$ $T_{f}^{*}$, we have

$$
\lim _{n \rightarrow \infty} \sum_{f \in F(n, \Lambda)}\left\|T_{f}^{*} h\right\|^{2}=\lim _{n \rightarrow \infty} \sum_{f \in F(n, \Lambda)}\left\|V_{f}^{*} h\right\|^{2}=0 \quad \text { for any } h \in \mathscr{H} .
$$

Conversely, assume that (2.2) holds. We claim that

$$
\lim _{n \rightarrow \infty} \sum_{f \in F(n, \Lambda)}\left\|V_{f}^{*} k\right\|^{2}=0 \quad \text { for any } k \in \mathscr{K}=\bigvee_{f \in \mathscr{F}} V_{f} \mathscr{H}
$$

By (2.2) we have

$$
\lim _{n \rightarrow \infty} \sum_{f \in F(n, \Lambda)}\left\|V_{f}^{*} h\right\|^{2}=0 \quad(h \in \mathscr{H}) .
$$


For each $k \in \bigvee_{f \in \mathscr{F} ; f \neq 0} V_{f} \mathscr{H}$ and any $\varepsilon>0$, there exists

$$
k_{\varepsilon}=\sum_{g \in \mathscr{F} ; g \neq 0}^{\prime} V_{g} h_{g} \quad\left(h_{g} \in \mathscr{H}\right)
$$

such that $\left\|k-k_{\varepsilon}\right\|<\varepsilon$. (Here $\sum^{\prime}$ stands for a finite sum.)

Since the isometries $V_{\lambda}(\lambda \in \Lambda)$ have orthogonal final spaces, it follows that

$$
\lim _{n \rightarrow \infty} \sum_{f \in F(n, \Lambda)}\left\|V_{f}^{*} k\right\|^{2}=\lim _{n \rightarrow \infty} \sum_{f \in F(n, \Lambda)}\left\|V_{f}^{*}\left(k-k_{\varepsilon}\right)\right\|^{2} \leq\left\|k-k_{\varepsilon}\right\|^{2}<\varepsilon^{2},
$$

for any $\varepsilon>0$. Thus, (2.3) holds and by Remark 1.4 we have that $\mathscr{V}$ is pure. This completes the proof.

Corollary 2.4. If $\sum_{\lambda \in \Lambda} T_{\lambda} T_{\lambda}^{*} \leq r I_{\mathscr{H}}, r<1$, then the minimal isometric dilation of $\mathscr{T}=\left\{T_{\lambda}\right\}_{\lambda \in \Lambda}$ is pure.

Now let us establish when the minimal isometric dilation $\mathscr{V}=\left\{V_{\lambda}\right\}_{\lambda \in \Lambda}$ cannot contain a $\Lambda$-orthogonal shift. The notations being the same as above we have

Proposition 2.5. $\sum_{\lambda \in \Lambda} V_{\lambda} V_{\lambda}^{*}=I_{\mathscr{H}}$ if and only if $\sum_{\lambda \in \Lambda} T_{\lambda} T_{\lambda}^{*}=I_{\mathscr{H}}$.

Proof. $(\Rightarrow)$ Since $\left.V_{\lambda}^{*}\right|_{\mathscr{H}}=T_{\lambda}^{*}(\lambda \in \Lambda)$ it follows that $\sum_{\lambda \in \Lambda} T_{\lambda} T_{\lambda}^{*} h=h$ $(h \in \mathscr{H})$.

$(\Leftarrow)$ If $\sum_{\lambda \in \Lambda} T_{\lambda} T_{\lambda}^{*}=I_{\mathscr{H}}$ then $\sum_{f \in F(n, \Lambda)}\left\|T_{f}^{*} h\right\|^{2}=\|h\|^{2}$ for any $n \in \mathbf{N}$ and $h \in \mathscr{H}$. Taking into account Theorem 1.3 let us assume that there exists $k \in \mathscr{K} \ominus\left(\bigoplus_{\lambda \in \Lambda} V_{\lambda} \mathscr{K}\right), k \neq 0$. Using Remark 1.4 it follows that

$$
\lim _{n \rightarrow \infty} \sum_{f \in F(n, \Lambda)}\left\|V_{f}^{*} k\right\|^{2}=0 .
$$

On the other hand, since

$$
\mathscr{K}=\mathscr{H} \vee\left(\bigvee_{\substack{f \in \mathscr{F} \\ f \neq 0}} V_{f} \mathscr{H}\right) \text { and } \bigvee_{\substack{f \in \mathscr{F} \\ f \neq 0}} V_{f} \mathscr{H} \subset \bigoplus_{\lambda \in \Lambda} V_{\lambda} \mathscr{K}
$$

it follows that $k \in \mathscr{H}$ and by (2.4) that $\lim _{n \rightarrow \infty} \sum_{f \in F(n, \Lambda)}\left\|T_{f}^{*} k\right\|^{2}=0$, contradiction. Thus we have $\sum_{\lambda \in \Lambda} V_{\lambda} V_{\lambda}^{*}=I_{\mathscr{K}}$ and the proof is complete.

Dropping out the minimality condition in the definition of the isometric dilation of a sequence $\mathscr{T}=\left\{T_{\lambda}\right\}_{\lambda \in \Lambda}$, we can prove the following.

Proposition 2.6. For any sequence $\mathscr{T}=\left\{T_{\lambda}\right\}_{\lambda \in \Lambda}$ of operators on a Hilbert space $\mathscr{H}$ such that $\sum_{\lambda \in \Lambda} T_{\lambda} T_{\lambda}^{*} \leq I_{\mathscr{H}}$ there exists an isometric dilation $\mathscr{V}=\left\{V_{\lambda}\right\}_{\lambda \in \Lambda}$ on a Hilbert space $\mathscr{K} \supset \mathscr{H}$ such that $\sum_{\lambda \in \Lambda} V_{\lambda} V_{\lambda}^{*}=I_{\mathscr{K}}$.

Proof. Taking into account Theorems 2.1 and 1.3, we show, without loss of generality, that the $\Lambda$-orthogonal shift $\mathscr{S}=\left\{S_{\lambda}\right\}_{\lambda \in \Lambda}$ on $\mathscr{H}_{0}=l^{2}(\mathscr{F}, \mathscr{E}) \quad(\mathscr{E}$ is 
a Hilbert space) can be extended to a sequence $\mathscr{V}=\left\{V_{\lambda}\right\}_{\lambda \in \Lambda}$ of isometries on a Hilbert space $\mathscr{K}_{0} \supset \mathscr{H}_{0}$ such that

$$
\sum_{\lambda \in \Lambda} V_{\lambda} V_{\lambda}^{*}=I_{\mathscr{H}_{0}} \quad \text { and }\left.\quad V_{\lambda}\right|_{\mathscr{H}_{0}}=S_{\lambda} \quad(\lambda \in \Lambda) .
$$

Consider the Hilbert space

$$
\mathscr{K}=\left[l^{2}(\mathscr{F}, \mathscr{E}) \ominus \mathscr{E}\right] \oplus l^{2}(\mathscr{F}, \mathscr{E}) .
$$

We embed $l^{2}(\mathscr{F}, \mathscr{E})$ into $\mathscr{K}$ by identifying the element $\left\{e_{f}\right\}_{f \in \mathscr{F}} \in l^{2}(\mathscr{F}, \mathscr{E})$ with the element $0 \oplus\left\{e_{f}\right\}_{f \in \mathscr{F}} \in \mathscr{K}$.

Let us define the isometries $V_{\lambda}(\lambda \in \Lambda)$ on $\mathscr{K}$. For $\lambda \geq 2$ we set $V_{\lambda}=$ $S_{\left.\lambda\right|^{2}(\mathscr{F}, \mathscr{E}) \Theta \mathcal{E}} \oplus S_{\lambda}$.

Consider the countable set

$$
\mathscr{F}^{\prime}=\{f \in \mathscr{F} \backslash F(1, \Lambda): f(1)=1\} \cup F(1, \Lambda) \cup\{0\}
$$

and a one-to-one map $\gamma: \mathscr{F} \backslash\{0\} \rightarrow \mathscr{F}^{\prime}$.

For $\left\{e_{f}^{*}\right\}_{f \in \mathscr{F} \backslash\{0\}} \oplus\left\{e_{f}\right\}_{f \in \mathscr{F}} \in \mathscr{K}$ the isometry $V_{1}$ is defined as follows

$$
\begin{gathered}
V_{1}\left(0 \oplus\left\{e_{f}\right\}_{f \in \mathscr{F}}\right)=0 \oplus S_{1}\left(\left\{e_{f}\right\}_{f \in \mathscr{F}}\right), \\
V_{1}\left(\left\{e_{f}^{*}\right\}_{f \in \mathscr{F} \backslash\{0\}} \oplus 0\right)=\left\{e_{g}^{\prime *}\right\}_{g \in \mathscr{F} \backslash\{0\}} \oplus\left\{e_{g}^{\prime}\right\}_{g \in \mathscr{F}},
\end{gathered}
$$

where

$$
e_{g}^{\prime *}= \begin{cases}e_{f}^{*} & \text { if } g=\gamma(f), \\ 0 & \text { otherwise }\end{cases}
$$

and

$$
e_{0}^{\prime}=e_{f}^{*} \quad \text { if } \gamma(f)=0, \quad e_{g}^{\prime}=0 \quad \text { if } g \in \mathscr{F} \backslash\{0\} .
$$

Now it is easy to see that the relations (2.5) hold.

Following the classification of contractions from [8] we give, in what follows, a classification of the sequences of contractions.

Let $\mathscr{T}=\left\{T_{\lambda}\right\}_{\lambda \in \Lambda}$ on a Hilbert space $\mathscr{H}$ such that $\sum_{\lambda \in \Lambda} T_{\lambda} T_{\lambda}^{*} \leq I_{\mathscr{H}}$.

Consider the following subspace of $\mathscr{H}$ :

$$
\begin{aligned}
& \mathscr{H}_{0}=\left\{h \in \mathscr{H}: \lim _{n \rightarrow \infty} \sum_{f \in F(n, \Lambda)}\left\|T_{f}^{*} h\right\|^{2}=0\right\}, \\
& \mathscr{H}_{1}=\left\{h \in \mathscr{H}: \sum_{f \in F(n, \Lambda)}\left\|T_{f}^{*} h\right\|^{2}=\|h\|^{2} \text { for any } n \in \mathbf{N}\right\} .
\end{aligned}
$$

Remark 2.7. The subspaces $\mathscr{H}_{0}$ and $\mathscr{H}_{1}$ are orthogonal and invariant for each operator $T_{\lambda}^{*}(\lambda \in \Lambda)$.

Proof. Taking into account Theorem 2.1, 1.3 and Remark 1.4 the proof is immediately.

Thus, the Hilbert space $\mathscr{H}$ decomposes into an orthogonal sum $\mathscr{H}=\mathscr{H}_{0} \oplus$ $\mathscr{H}_{1} \oplus \mathscr{H}_{2}$. 
For each $k \in\{0,1,2\}$ we shall denote by $C^{(k)}$ (respectively $C_{(k)}$ ) the set of all sequences $\mathscr{T}=\left\{T_{\lambda}\right\}_{\lambda \in \Lambda}$ on $\mathscr{H}$ for which we have $\mathscr{H}_{k}=\{0\}$ (respectively $\left.\mathscr{H}=\mathscr{H}_{k}\right)$.

Let us mention that $\mathscr{H}_{1}$ is the largest subspace in $\mathscr{H}$ on which the matrix

$$
\left[\begin{array}{c}
T_{1}^{*} \\
T_{2}^{*} \\
\vdots
\end{array}\right]
$$

acts isometrically.

Consequently, a sequence $\mathscr{T} \in C^{(1)}$ will be also called completely noncoisometric (c.n.c).

In the particular case when $\mathscr{T}=\{T\} \quad(\|T\| \leq 1)$ we have that $\mathscr{T} \in C^{(1)}$ if and only if $T^{*}$ is completely nonisometric, that is, if there is no nonzero invariant subspace for $T^{*}$ on which $T^{*}$ is an isometry.

We continue this section with the study of the geometric structure of the space of the minimal isometric dilation.

For this, let $\mathscr{T}=\left\{T_{\lambda}\right\}_{\lambda \in \Lambda}$ be a sequence of operators on a Hilbert space $\mathscr{H}$ such that $\sum_{\lambda \in \Lambda} T_{\lambda} T_{\lambda}^{*} \leq I_{\mathscr{H}}$ and $\mathscr{V}=\left\{V_{\lambda}\right\}_{\lambda \in \Lambda}$ be the minimal isometric dilation of $\mathscr{T}$ on the Hilbert space $\mathscr{K}=\mathscr{H} \oplus l^{2}(\mathscr{F}, \mathscr{D})$ (see Theorem 2.1).

Considering the subspaces of $\mathscr{K}$

$$
\mathscr{L}=\bigvee_{\lambda \in \Lambda}\left(V_{\lambda}-T_{\lambda}\right) \mathscr{H} \quad \text { and } \quad \mathscr{L}_{*}=\overline{\left(I_{\mathscr{H}}-\sum_{\lambda \in \Lambda} V_{\lambda} T_{\lambda}^{*}\right) \mathscr{H}}
$$

we can generalize some of the results from [ 8 , Chapter II, $\S \S 1,2]$ concerning the geometric structure of the space of the minimal isometric dilation.

Theorem 2.8. (i) The subspaces $\mathscr{L}$ and $\mathscr{L}_{*}$ are wandering subspaces for $\mathscr{V}$ and

$$
\operatorname{dim} \mathscr{L}=\operatorname{dim} \mathscr{D} ; \quad \operatorname{dim} \mathscr{L}_{*}=\operatorname{dim} \mathscr{D}_{*} .
$$

(ii) The space $\mathscr{K}$ can be decomposed as follows:

$$
\mathscr{K}=\mathscr{R} \oplus M_{\mathscr{F}}\left(\mathscr{L}_{*}\right)=\mathscr{H} \oplus M_{\mathscr{F}}(\mathscr{L}) \text {, }
$$

and the subspace $\mathscr{R}$ reduces each operator $V_{\lambda}(\lambda \in \Lambda)$.

(iii) $\mathscr{L} \cap \mathscr{L}_{*}=0$.

(iv) The subspace $\mathscr{R}$ reduces to $\{0\}$ if and only if $\mathscr{T} \in C_{(0)}$.

Proof. The Wold decomposition (see Theorem 1.3) for the minimal isometric dilation $\mathscr{V}$ on the space $\mathscr{K}=\mathscr{H} \oplus l^{2}(\mathscr{F}, \mathscr{D})$ gives $\mathscr{K}=\mathscr{R} \oplus M_{\mathscr{F}}\left(\mathscr{L}_{*}^{\prime}\right)$, where $\mathscr{R}=\bigcap_{n=0}^{\infty}\left[\bigoplus_{f \in F(n, \Lambda)} V_{f} \mathscr{K}\right]$ reduces each operator $V_{\lambda}(\lambda \in \Lambda)$ and $\mathscr{L}_{*}^{\prime}=\mathscr{K} \ominus\left(\bigoplus_{\lambda \in \Lambda} V_{\lambda} \mathscr{K}\right)$ is a wandering subspace for $\mathscr{V}$. by

It is easy to see that $\mathscr{L}_{*}^{\prime}=\mathscr{L}_{*}$ and that the operator $\Phi_{*}: \mathscr{L}_{*} \rightarrow \mathscr{D}_{*}$ defined

$$
\Phi_{*}\left(I_{\mathscr{H}}-\sum_{\lambda \in \Lambda} V_{\lambda} T_{\lambda}^{*}\right) h=D_{*} h \quad(h \in \mathscr{H})
$$


is unitary. Hence it follows that $\operatorname{dim} \mathscr{L}_{*}=\operatorname{dim} \mathscr{D}_{*}$. Equation (2.1) yields

$$
\sum_{\lambda \in \Lambda}\left(V_{\lambda}-T_{\lambda}\right) h_{\lambda}=0 \oplus D\left(\left(h_{\lambda}\right)_{\lambda \in \Lambda}\right) \quad \text { for }\left(h_{\lambda}\right)_{\lambda \in \Lambda} \in \bigoplus_{\lambda \in \Lambda} \mathscr{H}_{\lambda}
$$

$\left(\mathscr{H}_{\lambda}\right.$ is a copy of $\left.\mathscr{H}\right)$.

By this relation we deduce that there exists a unitary operator $\Phi: \mathscr{L} \rightarrow \mathscr{D}$ defined by equation

$$
\Phi\left(\sum_{\lambda \in \Lambda}\left(V_{\lambda}-T_{\lambda}\right) h_{\lambda}\right)=D\left(\left(h_{\lambda}\right)_{\lambda \in \Lambda}\right)
$$

and hence that $\operatorname{dim} \mathscr{L}=\operatorname{dim} \mathscr{D}$.

The fact that $\mathscr{L}$ is a wandering subspace for $\mathscr{V}$ and that $\mathscr{H} \perp M_{\mathscr{F}}(\mathscr{L})$ follows from the form of the isometries $V_{\lambda}(\lambda \in \Lambda)$ defined by (2.1).

Taking into account the minimality of $\mathscr{\mathscr { K }}$ it follows that $\mathscr{K}=\mathscr{H} \oplus M_{\mathscr{F}}(\mathscr{L})$.

Let us now show that $\mathscr{L} \cap \mathscr{L}_{*}=0$. First we need to prove that

$$
\mathscr{L}_{*} \oplus\left(\bigoplus_{\lambda \in \Lambda} V_{\lambda} \mathscr{H}\right)=\mathscr{H} \oplus \mathscr{L}
$$

This follows from the fact that, for an element $u \in \mathscr{K}$, the possibility of a representation of the form

$$
u=\left(I_{\mathscr{H}}-\sum_{\lambda \in \Lambda} V_{\lambda} T_{\lambda}^{*}\right) h_{0}+\sum_{\lambda \in \Lambda} V_{\lambda} h_{\lambda}, \quad h_{0} \in \mathscr{H},\left(h_{\lambda}\right)_{\lambda \in \Lambda} \in \bigoplus_{\lambda \in \Lambda} \mathscr{H}_{\lambda},
$$

is equivalent to the possibility of a representation of the form

$$
u=h^{(0)}+\sum_{\lambda \in \Lambda}\left(V_{\lambda}-T_{\lambda}\right) h^{(\lambda)}, \quad h^{(0)} \in \mathscr{H},\left(h^{(\lambda)}\right)_{\lambda \in \Lambda} \in \bigoplus \mathscr{H}_{\lambda} .
$$

Indeed, we have only to set

$$
h_{0}=h^{(0)}-\sum_{\lambda \in \Lambda} T_{\lambda} h^{(\lambda)}, \quad h_{\lambda}=T_{\lambda}^{*} h^{(0)}+h^{(\lambda)}
$$

and, conversely,

$$
h^{(0)}=\sum_{\lambda \in \Lambda} T_{\lambda} h_{\lambda}+\left(I_{\mathscr{H}}-\sum_{\lambda \in \Lambda} T_{\lambda} T_{\lambda}^{*}\right) h_{0}, \quad h^{(\lambda)}=h_{\lambda}-T_{\lambda}^{*} h_{0} .
$$

Thus (2.8) holds. On the other hand, since

$$
\mathscr{L} \subset\left(\bigoplus_{\lambda \in \Lambda} V_{\lambda} \mathscr{H}\right) \vee \mathscr{H} \quad \text { and } \bigoplus_{i \in \Lambda} V_{\lambda} \mathscr{H} \subset \mathscr{L} \oplus \mathscr{H}
$$

we have that $\mathscr{H} \vee\left(\bigoplus_{i \in \Lambda} V_{\lambda} \mathscr{H}\right)=\mathscr{H} \oplus \mathscr{L}$. This relation and (2.8) show that $\mathscr{L} \cap \mathscr{L}_{*}=\{0\}$.

The statement (iv) is contained in Proposition 2.3. The proof is complete. 
Propostion 2.9. For every sequence $\mathscr{T}=\left\{T_{\lambda}\right\}_{\lambda \in \Lambda}$ of operators on $\mathscr{H}$ and for its minimal isometric dilation $\mathscr{V}=\left\{V_{\lambda}\right\}_{\lambda \in \Lambda}$ on $\mathscr{K}$, we have

$$
M_{\mathscr{F}}(\mathscr{L}) \vee M_{\mathscr{F}}\left(\mathscr{L}_{*}\right)=\mathscr{K} \ominus \mathscr{H}_{1}
$$

where $\mathscr{H}_{1}$ is given by (2.7).

In particular, if $\mathscr{T}$ is c.n.c., then

$$
M_{\mathscr{F}}(\mathscr{L}) \vee M_{\mathscr{F}}\left(\mathscr{L}_{*}\right)=\mathscr{K}
$$

Proof. Taking into account Theorem 2.8 and that $\mathscr{H}_{1} \subset \mathscr{R}$ it follows that $\mathscr{H}_{1} \perp M_{\mathscr{F}}(\mathscr{L}) \vee M_{\mathscr{F}}\left(\mathscr{L}_{*}\right)$.

Now let $k \in \mathscr{K}$ be such that $k \perp M_{\mathscr{T}}(\mathscr{L})$ and $k \perp M_{\mathscr{F}}\left(\mathscr{L}_{*}\right)$.

From the same theorem it follows that $k \in \mathscr{H}$ and $k \perp V_{f} \mathscr{L}_{*}$ for every $f \in \mathscr{F}$. Hence we have

$$
0=\left(k, V_{f}\left(I_{\mathscr{H}}-\sum_{\lambda \in \Lambda} V_{\lambda} T_{\lambda}^{*}\right) h\right)=\left(T_{f}^{*} k, h\right)-\sum_{\lambda \in \Lambda}\left(T_{\lambda}^{*} T_{f}^{*} k, T_{\lambda}^{*} h\right)
$$

for every $h \in \mathscr{H}$.

Choosing $h=T_{f}^{*} k \quad(f \in \mathscr{F})$ we obtain

$$
\left\|T_{f} k\right\|^{2}=\sum_{\lambda \in \Lambda}\left\|T_{\lambda}^{*} T_{f}^{*} k\right\|^{2}
$$

for any $f \in \mathscr{F}$.

Hence we deduce

$$
\sum_{g \in F(n, \Lambda)}\left\|T_{g}^{*} k\right\|^{2}=\|k\|^{2}
$$

for any $n \in \mathbf{N}$. We conclude that $k \in \mathscr{H}_{1}$. Conversely, for every $k \in \mathscr{H}_{1}$ it is easy to see that $k \perp M_{\mathscr{F}}(\mathscr{L}) \vee M_{\mathscr{F}}\left(\mathscr{L}_{*}\right)$. The relation (2.10) follows because for $\mathscr{T}$ c.n.c. we have $\mathscr{H}_{1}=\{0\}$.

The last aim of this section is to generalize some of the results from [8, Chapter II, §3]. Throughout $\mathscr{V}=\left\{V_{\lambda}\right\}_{\lambda \in \Lambda}$ is the minimal isometric dilation of $\mathscr{T}=\left\{T_{\lambda}\right\}_{\lambda \in \Lambda}$. The space of the minimal isometric dilation is

$$
\mathscr{K}=\mathscr{R} \oplus M_{\mathscr{F}}\left(\mathscr{L}_{*}\right)=\mathscr{H} \oplus l^{2}(\mathscr{F}, \mathscr{D}) .
$$

Proposition 2.10. For every $h \in \mathscr{H}$ we have

$$
P_{\mathscr{h}} h=\lim _{n \rightarrow \infty} \sum_{f \in F(n, \Lambda)} V_{f} T_{f}^{*} h
$$

and consequently

$$
\left\|P_{\mathscr{H}} h\right\|^{2}=\lim _{n \rightarrow \infty} \sum_{f \in F(n, \Lambda)}\left\|T_{f}^{*} h\right\|^{2}
$$

where $P_{\mathscr{H}}$ denotes the orthogonal projection of $\mathscr{K}$ into $\mathscr{H}$. 
Proof. An easy computation shows that

$$
\begin{aligned}
& \left\|\sum_{f \in F(n+1, \Lambda)} V_{f} T_{f}^{*} h-\sum_{f \in F(n, \Lambda)} V_{f} T_{f}^{*} h\right\|^{2} \\
& \quad=\sum_{f \in F(n+1, \Lambda)}\left\|T_{f}^{*} h\right\|^{2}-\sum_{f \in F(n, \Lambda)}\left\|T_{f}^{*} h\right\|^{2} \leq 0
\end{aligned}
$$

for every $n \in \mathbf{N}$. This implies the convergence of $\left\{\sum_{f \in F(n, \Lambda)} V_{f} T_{f}^{*} h\right\}_{n=1}^{\infty}$ the sequence in $\mathscr{K}$. Setting

$$
k=\lim _{n \rightarrow \infty} \sum_{f \in F(n, \Lambda)} V_{f} T_{f}^{*} h,
$$

let us show that $k=P_{\mathscr{R}} h$, i.e. $k \perp M_{\mathscr{F}}\left(\mathscr{L}_{*}\right)$ and $h-k \in M_{\mathscr{F}}\left(\mathscr{L}_{*}\right)$.

Since for every $g \in \mathscr{F}$ there exists $n_{0} \in N$ such that

$$
\sum_{f \in F(n, \Lambda)} V_{f} T_{f}^{*} h \perp V_{g} \mathscr{L}_{*}
$$

for any $n \geq n_{0}$, it follows that $k \perp M_{\mathscr{F}}\left(\mathscr{L}_{*}\right)$.

On the other hand we have

$$
\begin{aligned}
h-\sum_{f \in F(n, \Lambda)} V_{f} T_{f}^{*} h= & \left(I_{\mathscr{H}}-\sum_{\lambda \in \Lambda} V_{\lambda} T_{\lambda}^{*}\right) h+\sum_{f \in F(1, \Lambda)} V_{f}\left(I_{\mathscr{H}}-\sum_{\lambda \in \Lambda} V_{\lambda} T_{\lambda}^{*}\right) T_{f}^{*} h \\
& +\cdots+\sum_{f \in F(n-1, \Lambda)} V_{g}\left(I_{\mathscr{H}}-\sum_{\lambda \in \Lambda} V_{\lambda} T_{\lambda}^{*}\right) T_{g}^{*} h \in M_{\mathscr{F}}\left(\mathscr{L}_{*}\right)
\end{aligned}
$$

and therefore

$$
h-k=\lim _{n \rightarrow \infty}\left(h-\sum_{f \in F(n, \Lambda)} V_{f} T_{f}^{*} h\right) \in M_{\mathscr{F}}\left(\mathscr{L}_{*}\right) .
$$

This ends the proof.

Proposition 2.11. Let $\mathscr{T}=\left\{T_{\lambda}\right\}_{\lambda \in \Lambda}$ be a sequence of operators on $\mathscr{H}$ such that the matrix $\left[T_{1}, T_{2}, \ldots\right]$ is an injection. Then $\overline{P_{\mathscr{R}} \mathscr{H}}=\mathscr{R}$.

Proof. Let us suppose that there exists $k \in \mathscr{R}, k \neq 0$ such that $k \perp P_{\mathscr{R}} \mathscr{H}$, or equivalently, such that $k \perp M_{\mathscr{F}}\left(\mathscr{L}_{*}\right)$ and $k \perp \mathscr{H}$.

By Theorem 2.8 we have $\mathscr{K}=\mathscr{H} \oplus M_{\mathscr{F}}(\mathscr{L})$. It follows that $k \in M_{\mathscr{F}}(\mathscr{L})$ and hence $k=\sum_{f \in \mathscr{F}} V_{f} l_{f}$ where $l_{f} \in \mathscr{L}(f \in \mathscr{F})$ and $\sum_{f \in \mathscr{F}}\left\|l_{f}\right\|^{2}<\infty$. Since $k \neq 0$ there exists $f_{0} \in \mathscr{F}$, such that $V_{f_{0}} l_{f_{0}} \neq 0$ and

$$
V_{f_{0}}^{*} k=l_{f_{0}}+\sum_{\substack{g \in \mathscr{F} \\ g \neq 0}} V_{g} l_{g}^{\prime} \quad\left(l_{g}^{\prime} \in \mathscr{L}\right) .
$$

One can easily show that for every $g \in \mathscr{F}, g \neq 0, V_{g} \mathscr{L} \perp \mathscr{L}_{*}$. Since $V_{f_{0}}^{*} k \perp \mathscr{L}_{*}$ it follows that $l_{f_{0}} \perp \mathscr{L}_{*}$. By the relation (2.8) we deduce that $l_{f_{0}} \in \bigoplus_{\lambda \in \Lambda} V_{\lambda} \mathscr{H}$. 
Therefore, there exists a nonzero $\bigoplus_{\lambda \in \Lambda} h_{\lambda} \in \bigoplus_{\lambda \in \Lambda} \mathscr{H}_{\lambda}$ such that $l_{f_{0}}=$ $\sum_{\lambda \in \Lambda} V_{\lambda} h_{\lambda}$. Since $\mathscr{L} \perp \mathscr{H}$, it follows that $\sum_{\lambda \in \Lambda} T_{\lambda} h_{\lambda}=0$ which is a contradiction with the hypothesis.

Thus $\overline{P_{\mathscr{R}} \mathscr{H}}=\mathscr{R}$ and the proof is complete.

For each $\lambda \in \Lambda$ let us denote by $R_{\lambda}$ the operator $\left.V_{\lambda}\right|_{\mathscr{R}}$. Taking into account the Wold decomposition (Theorem 1.3) we have $\sum_{\lambda \in \Lambda} R_{\lambda} R_{\lambda}^{*}=I_{\mathscr{R}}$.

The following theorem is a generalization of Proposition 3.5 in [8, Chapter II].

Proposition 2.12. Let $\mathscr{T}=\left\{T_{\lambda}\right\}_{\lambda \in \Lambda}$ a sequence of operators on $\mathscr{H}$ such that $\mathscr{T} \in C^{(0)}$ and the matrix $\left[T_{1}, T_{2}, \ldots\right]$ is an injective contraction.

Then $\mathscr{T}$ is quasi-similar to $\left\{R_{\lambda}\right\}_{\lambda \in \Lambda}$, i.e., there exists a quasi-affinity $Y$ from $\mathscr{R}$ to $\mathscr{H}$ such that $T_{\lambda} Y=Y R_{\lambda}$ for every $\lambda \in \Lambda$.

Proof. According to Proposition 2.10 we have

$$
\begin{aligned}
V_{\lambda}^{*} P_{\mathscr{R}} h & =\lim _{n \rightarrow \infty} \sum_{f \in F(n, \Lambda)} V_{\lambda}^{*} V_{f} T_{f}^{*} h \\
& =\lim _{n \rightarrow \infty} \sum_{g \in F(n-1, \Lambda)} V_{g} T_{g}^{*} T_{\lambda}^{*} h=P_{\mathscr{R}} T_{\lambda}^{*} h
\end{aligned}
$$

for all $h \in \mathscr{H}$ and each $\lambda \in \Lambda$.

Setting $X=\left.P_{\mathscr{R}}\right|_{\mathscr{H}}$ it follows that $R_{\lambda}^{*} X=X T_{\lambda}^{*}$ for every $\lambda \in \Lambda$. Let us show that $X$ is a quasi-affinity.

Since $\mathscr{T} \in C^{(0)}$ we have that

$$
\lim _{n \rightarrow \infty} \sum_{f \in F(n, \Lambda)}\left\|T_{f}^{*} h\right\|^{2}=0 \quad \text { for every nonzero } h \in \mathscr{H} .
$$

By Proposition 2.10 we deduce that $P_{\mathscr{R}} h \neq 0$ for every nonzero $h \in \mathscr{H}$, i.e., $X$ is an injection.

On the other hand, Proposition 2.11 shows that $\overline{X \mathscr{H}}=\mathscr{R}$.

If we take $Y=X^{*}$, this finishes the proof.

In this section we extend the Sz.-Nagy-Foias lifting theorem $[7,8,1,4]$ to our setting.

Let $\mathscr{T}=\left\{T_{\lambda}\right\}_{\lambda \in \Lambda}$ be a sequence of operators on $\mathscr{H}$ with $\sum_{\lambda \in \Lambda} T_{\lambda} T_{\lambda}^{*} \leq I_{\mathscr{H}}$ and $\mathscr{V}=\left\{V_{\lambda}\right\}_{\lambda \in \Lambda}$ be the minimal isometric dilation of the Hilbert space $\mathscr{K}=$ $\mathscr{H} \oplus l^{2}(\mathscr{F}, \mathscr{D})$ (see Theorem 2.1).

Consider the following subspaces of $\mathscr{K}$

$$
\mathscr{H}_{1}=\mathscr{H} \vee\left(\bigvee_{f \in F(1, \Lambda)} V_{f} \mathscr{H}\right)
$$


and

$$
\mathscr{H}_{n}=\mathscr{H}_{n-1} \vee\left(\bigvee_{f \in F(1, \Lambda)} V_{f} \mathscr{H}_{n-1}\right) \text { for } n \geq 2 .
$$

Note that $\mathscr{H}_{n} \subset \mathscr{H}_{n+1}$ and that all the space $\mathscr{H}_{n}(n \geq 1)$ are invariant for each operator $V_{\lambda}^{*}(\lambda \in \Lambda)$.

As in $[7,8,1,4]$ the $n$-stepped dilation of $\mathscr{T}$ is the sequence $\mathscr{T}_{n}=\left\{\left(T_{\lambda}\right)_{n}\right\}_{\lambda \in \Lambda}$ of operators defined by $\left(T_{\lambda}\right)_{n}^{*}=\left.V_{\lambda}^{*}\right|_{\mathscr{H}_{n}}(n \geq 1, \lambda \in \Lambda)$.

One can easily show that $\mathscr{V}$ is the minimal isometric dilation on $\mathscr{T}_{n}$ and that $\mathscr{T}_{n+1}$ is the one-step dilation of $\mathscr{T}_{n}$.

Let us observe that $\mathscr{H}_{1}=\mathscr{H} \oplus \mathscr{D}$ and

$$
\mathscr{H}_{n}=\mathscr{H} \oplus \mathscr{D} \oplus\left(\bigoplus_{f \in F(1, \Lambda)} S_{f} \mathscr{D}\right) \oplus \cdots \oplus\left(\bigoplus_{f \in F(n-1, \Lambda)} S_{f} \mathscr{D}\right) \quad(n \geq 2)
$$

where $\mathscr{S}=\left\{S_{\lambda}\right\}_{\lambda \in \Lambda}$ is the $\Lambda$-orthogonal shift acting on $l^{2}(\mathscr{F}, \mathscr{D})$.

Now Lemma 2 and Theorem 3 in [4] can be easily extended to our setting. Thus, we omit the proofs in what follows.

Lemma 3.1. Let $P_{n}$ be the orthogonal projection from $\mathscr{K}$ into $\mathscr{H}_{n}$.

Then $\bigvee_{n \geq 1} \mathscr{H}_{n}=\mathscr{K}$ and for each $\lambda \in \Lambda$ we have

$$
\left(T_{\lambda}\right)_{n}^{*} P_{n} \rightarrow V_{\lambda}^{*} \quad \text { (strongly) as } n \rightarrow \infty .
$$

Let $\mathscr{T}^{\prime}=\left\{T_{\lambda}^{\prime}\right\}_{\lambda \in \Lambda}$ be another sequence of operators on a Hilbert space $\mathscr{H}^{\prime}$ with $\sum_{\lambda \in \Lambda} T_{\lambda}^{\prime} T_{\lambda}^{\prime *} \leq I_{\mathscr{H}}$, and $\mathscr{V}^{\prime}=\left\{V_{\lambda}^{\prime}\right\}_{\lambda \in \Lambda}$ be the minimal isometric dilation of $\mathscr{T}^{\prime}$ acting on the Hilbert space $\mathscr{K}^{\prime}=\mathscr{H}^{\prime} \oplus l^{2}\left(\mathscr{F}, \mathscr{D}^{\prime}\right)$.

Theorem 3.2. Let $A: \mathscr{H} \rightarrow \mathscr{H}^{\prime}$ be a contraction such that for each $\lambda \in \Lambda$ $T_{\lambda}^{\prime} A=A T_{\lambda}$. Then there exists a contraction $B: \mathscr{K} \rightarrow \mathscr{K}^{\prime}$ such that for each $\lambda \in \Lambda \quad V_{\lambda}^{\prime} B=B V_{\lambda}$ and $\left.B^{*}\right|_{\mathscr{H}^{\prime}}=A^{*}$.

\section{REFERENCES}

1. R. G. Douglas, P. S. Muhly and C. M. Pearcy, Lifting commuting operators, Michigan Math. J. 15 (1968), 385-395.

2. C. Foiaş, A remark on the universal model for contractions of G. C. Rota, Comm. Acad. R. P. Romane 13 (1963), 349-352.

3. A. E. Frazho, Models for noncommuting operators, J. Funct. Anal. 48 (1982).

4. Complements to models for noncommuting operators, J. Funct. Anal. 59 (1984), 445-461.

5. G. Popescu, Models for infinite sequences of noncommuting operators, INCREST preprint, no. $23 / 1986$.

6. J. J. Schaffer, On unitary dilations of contractions, Proc. Amer. Math. Soc. 6 (1955), 322.

7. B. Sz.-Nagy and C. Foiaş, Dilation des commutants, C. R. Acad. Sci. Paris Sér. A 266 (1968), 201-212.

8. __ Harmonic analysis on operators on Hilbert space, North-Holland, Amsterdam, 1970.

Department of Mathematics, INCREST, Bd. Pacil 220, 79622 Bucharest, Romania 